\title{
Blocking Performance for All Optical Wavelength Routed WDM Networks under Wavelength Conversions
}

\author{
Farouk E. El-Khamy ${ }^{\text {, }}$ Mohamed Nasr ${ }^{\text {ii }}$, Hossam M. H. Shalaby ${ }^{\text {iii }}$ and Hussein T. Mouftah ${ }^{\text {iv }}$ \\ ${ }^{i}$ Consultant with National Telecommunication Institute, Cairo, Egypt, e-mail: felkhamy@gmail.com \\ ${ }^{i i}$ Dept of Electronic \& Communication, Faculty of Engineering, Tanta University, Egypt \\ ${ }^{i i i}$ Dept. of Electrical Engineering, Faculty of Engineering, University of Alexandria, Egypt \\ ${ }^{i v}$ Canada Research Chair, Distinguished Univ. Professor and Professor, School of Info. Tech\& Eng., Ottawa
}

\begin{abstract}
Wavelength converters relax the wavelength continuity constrain in wavelength routed WDM all optical networks. Since wavelength converter is an expensive component with respect to other components in optical network researches are constrained in minimizing this coast keeping the blocking performance as optimum as we can. Sparse wavelength converters, limited range wavelength converters, shared node wavelength converters and node shared wavelength converters are examples used to optimize this problem. In this paper blocking performance optimization for convertible routers in WDM optical networks are examined using simulation. Simulation results show that full wavelength converters for large paths give significant enhancement in blocking performance than the non wavelength converter router path of the same number of nodes. wavelength converters depends on many factors such as network topology, number of wavelengths available, and traffic patterns

Keywords: Wavelength routing optical network, Wave division multiplexing, Wavelength Converters, Blocking performance.
\end{abstract}

\section{INTRODUCTION}

Wavelength-routed networks can effectively utilize the bandwidth of the optical fibres. Wavelength converters help to reduce the blocking probability of the network and enhance the fibre utilization. One of the primary design objectives of wavelength-routed all-optical networks is to minimize the blocking probability. In a network without wavelength converter nodes, it is required that data for a call arriving at the input port of a node on one wavelength has to be switched to an output port of the node at the same wavelength. To satisfy such a connection request for a call, it is necessary that the connection path which is setup from the source to the destination has a free wavelength on its entire links. This requirement is called the wavelength continuity constraint and results in increasing the probability of a call being blocked. On the other hand, it has been shown in [1] that wavelength converters improve the performance of wavelength-routed networks in terms of blocking probability. Wavelength-routed networks can effectively utilize the bandwidth of the optical fibres. Wavelength converters help to reduce the blocking probability of the network and enhance the fibre utilization [2]. In this paper we investigate the problem of wavelength Conversion in wavelength-routed networks comparing them with no wavelength conversion networks. We estimated the blocking probability when using both first fit wavelength assignment algorithm and random wavelength assignment algorithm.

\section{WAVELENGTH-CONVERTIBLE NODES NETWORKS}

Wavelength conversion technology and wavelength converters play an important role in enhancing fiber utilization and in reducing the overall call blocking probability of the network. Wavelength conversion eliminates the wavelength continuity constraint and thus improves the blocking performance significantly [3]. Since wavelength converters are still very expensive, much research focuses on sparse wavelength conversion and limited wavelength conversion. A wavelength convertible router (WCR) is a router capable of wavelength conversion, sparse wavelength conversion in which only some of the network nodes have the capability of wavelength Conversion, however if all the network nodes are WCRs this is referred to as full wavelength conversion. A Wavelength Converter is an optical device that can be used in an optical router, to convert the wavelength a channel is being carried on [2]. Without wavelength conversion, an incoming signal from port $P_{i}$ on, say, the wavelength $\lambda_{1}$ can be optically switched (without intermediate optoelectronic conversions) to any port $P_{i}$, but only on the wavelength $\lambda_{1}$. With wavelength conversion capability, this signal could be optically switched to any port $P_{i}$ on any wavelength $\lambda_{k}$ that is, wavelength conversion allows a clear optical channel to be carried on different wavelengths on different physical links. Different levels of wavelength conversion capability are possible. Figure 1 illustrates the differences for a single input and single output port situation; the case for multiple ports is more complicated but similar. Full wavelength conversion capability implies that any input wavelength may be converted to any other wavelength Limited wavelength conversion denotes that each input wavelength may be converted to any of a specific set of wavelengths, which is not the set of all wavelengths for at least one input wavelength. The advantage of wavelength conversion is that the virtual 
topology that can be implemented is less constrained, since the wavelength continuity constraint is removed. Thus wavelength use is more efficient. However, the use of converters increases cost, as well as the complexity of the problem. The cost increase can be minimized by using limited conversion rather than full conversion, and assuming a small number of converters rather than conversion capability in every node. But these assumptions introduce the problems of specifying the nature of the limited conversion and placement of converters in the network, which greatly increase the difficulty of topology design.

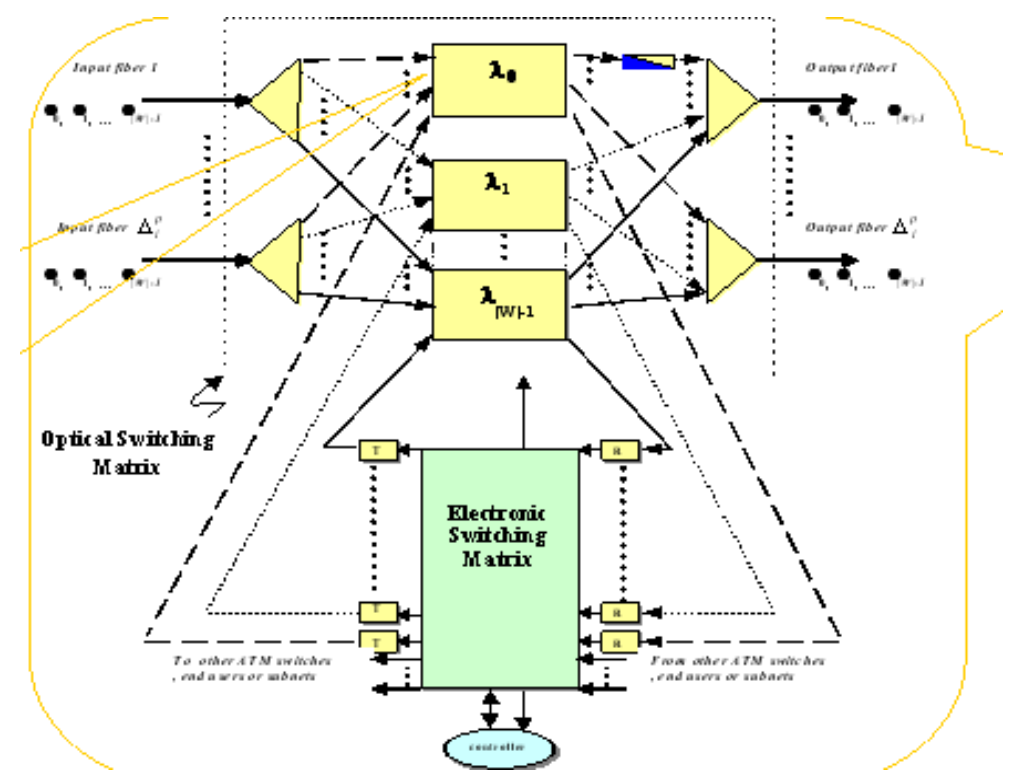

Figure 1. Wavelength Convertible Optical Switch.

\section{ANALYTICAL MODEL}

Our analytical model includes link traffic analysis and path-blocking analysis. The link traffic analysis consists of a set of equations that determine the traffic offered to each link (so called link-offered traffic) from the pathblocking probabilities. On the other hand, the path-blocking analysis consists of a set of equations that determine the path-blocking probabilities from the link-offered traffic. These two parts of analysis lead to a set of fixedpoint non-linear equations and they can be solved by iterative substitutions.

The overall blocking probability $P$ is the ratio of blocked traffic to the offered traffic. That is

$$
P=\frac{\sum_{a}\left(A^{a}-\overline{A^{a}}\right)}{\sum_{a} A^{a}} .
$$

The traffic for node pair $a$ can be carried on any of its provided paths. And the connection will be blocked if and only if it is blocked on all the $M_{a}$, number of paths. Given the assumption that these paths are link-disjoint, we can consider the blocking events on these paths to be independent with each other. So we can have

$$
\overline{A^{a}}=A^{a}\left(1-\prod_{t=1}^{M_{a}} B_{R_{a}}(t)\right) .
$$

From Eq. (1) and Eq. (2), we can simplify the overall blocking probability as

$$
P=\frac{\sum_{a} A^{a} \prod_{t=1}^{M_{a}} B_{R_{a}^{(t)}}}{\sum_{a} A^{a}} .
$$




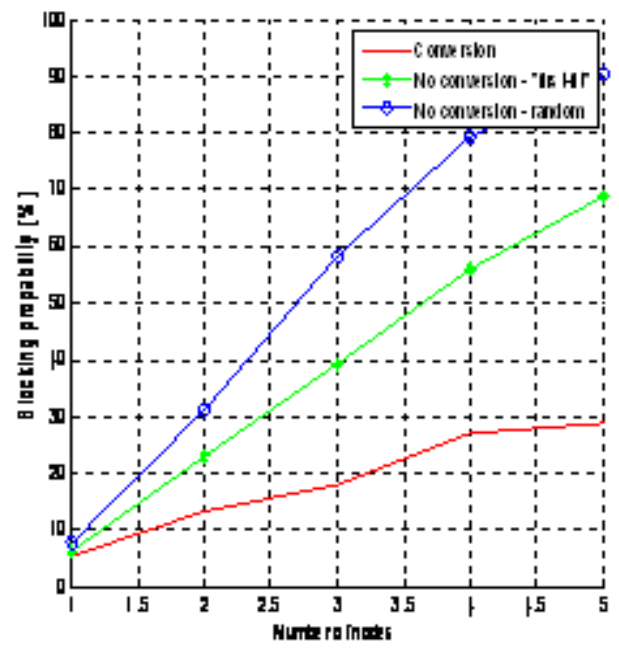

Fig. 2a. Number of nodes $=5$.

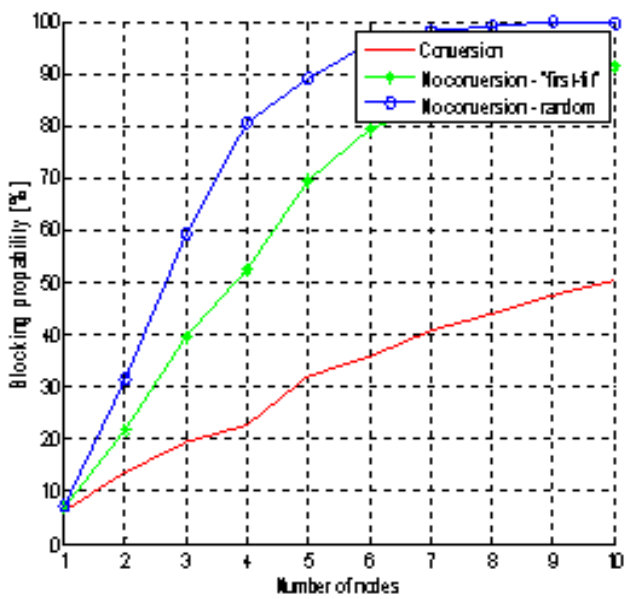

Fig. 2b. Number of nodes $=10$.

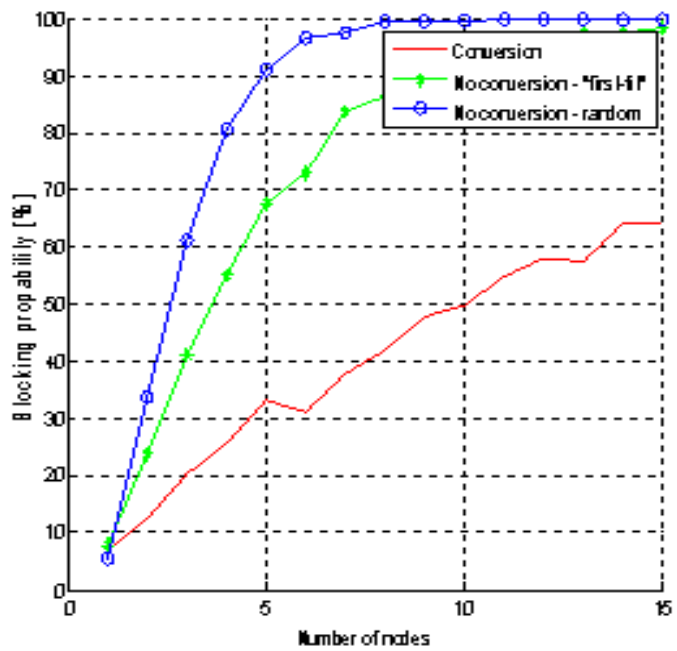

Fig. 2c. Number of nodes $=15$.

Figure. 2. Effect of varying number of nodes on the performance of the blocking in cases of no conversion and conversion using number of channels $=8$ and load of 5 Erlang.

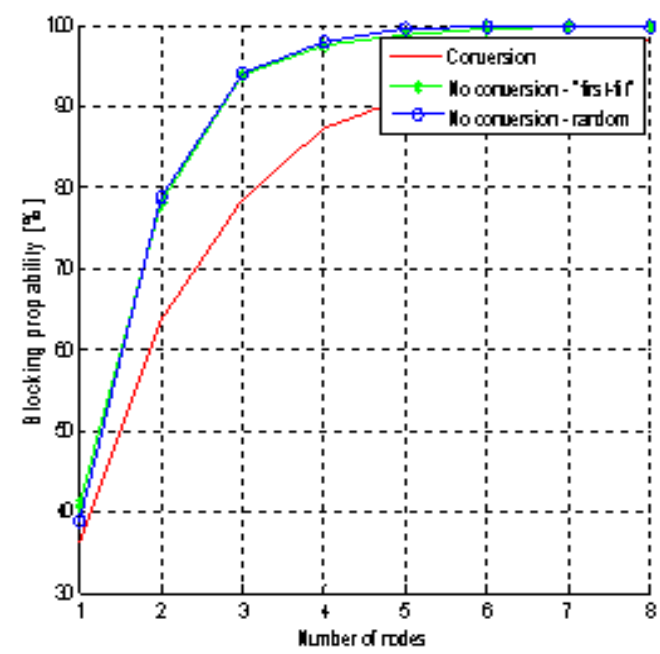

Fig. 3a. Number of wavelengths $=4$.

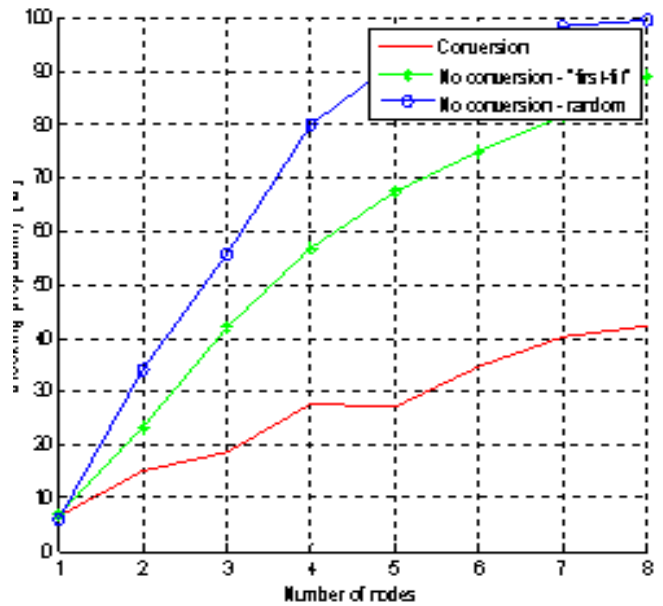

Fig. 3b. Number of Wavelengths $=8$.

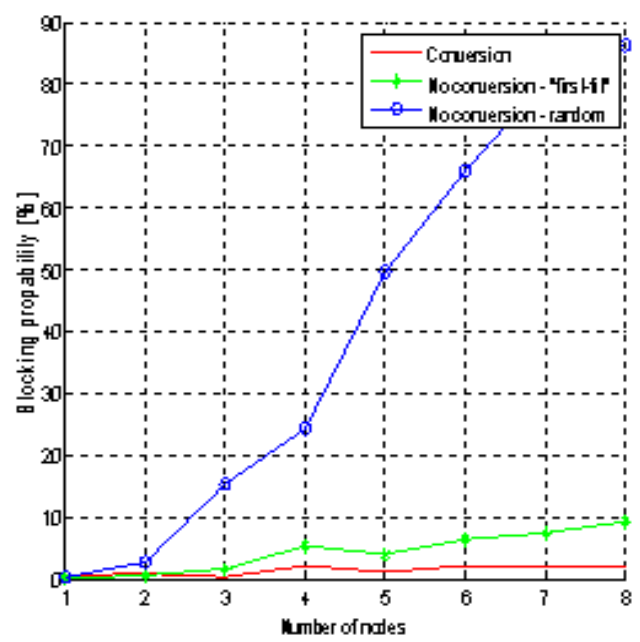

Fig. 3c. Number of wavelengths $=16$.

Figure 3. Effect of varying number of wavelengths on the performance of the blocking in cases of no conversion and conversion using number of nodes $=8$ and load of 5 Erlang. 


\section{BLOCKING PERFORMANCE SIMULATION RESULTS FOR WAVELENGTH CONVERTIBLE ROUTERS}

The simulation in this section has been carried out using Mat lab computer program with the help of an open source tool box called Blocking computations in WDM networks tool box [4]. In the first stage of this simulation, We check the effect of increasing the number of nodes keeping fixed traffic loads and number of channels; simulation has carried out for both full wavelength conversation case and no-wavelength conversation case. In the case of no wavelength conversion, we use two wavelength assignment approaches the first fit method and the random method for wavelength assignment. From Fig. 2, we notes that the blocking probability increases very fast by increasing the number of nodes, the full conversion case gives little enhancement in blocking performance than the no-conversation cases and no conversion using first fit wavelength assignment always gives better results than that of no-conversion using random wavelength assignment. In the second stage of this simulation, we vary the number of wavelength used keeping all other resources constant (eight nodes, five Erlang traffic load). As shown in Fig. 3 we assure that increasing number of wavelength used enhance the blocking performance, we show using full wavelength conversion with a large number of used wavelengths gives almost non blocked condition as shown in Fig. 3c.

\section{CONCLUSIONS}

The blocking performance with wavelength converters depends on many factors such as network topology, number of wavelengths available, and traffic patterns. For short paths there is no significant change in the blocking performance in case of full conversion case than that of no-conversion case but for long paths there is a large enhancement especially for the large traffic loads. Results show that, significant improvement in the blocking performance of the network is achieved when $50 \%$ of the nodes are equipped with wavelength converters using the same traffic load.

\section{REFERENCES}

[1] G. Rouskas and Harry Perros "A tutorial on optical networks", Advance Lectures on Networking: Networking 2002 Tutorials, Gregori et al. (Eds.), Springer LNCS 2497, pp. 155.

[2] Tarek S. El-Bawab “Optical Switching in Communications Networks” Book Optical switching chapter, Publisher Springer US ISBN 978-0-387-26141-6.

[3] B. Ramamurthy, B. Mukherjee, "Wavelength conversion in WDM networking," IEEE Journal on Selected Areas in Communications, vol. 16, no. 7, pp. 1061-1073, Sep. 1998.

[4] P. Pawelczak "WDM Network Blocking Computation Toolbox", MATLAB Central, http://www.mathworks.com/matlabcentral/fileexchange/4797. 